\title{
Lipopolysaccharide preconditioning induces robust protection against brain injury resulting from deep hypothermic circulatory arrest
}

Edward J. Hickey, MRCS, ${ }^{a}$ Xiaomang You, MD, ${ }^{a}$ Vassil Kaimaktchiev, MD, ${ }^{\mathrm{b}}$ Mary Stenzel-Poore, PhD, ${ }^{\mathrm{c}}$ and Ross M. Ungerleider, MD ${ }^{a}$

From the Departments of Pediatric Cardiac Surgery, ${ }^{\mathrm{a}}$ Neuropathology, ${ }^{\mathrm{b}}$ and Immunology, ${ }^{c}$ Oregon Health Sciences University, Portland, Ore.

Supported by generous grants from the Medical Research Foundation of Oregon and the Children's Heart Foundation, which have funded both this study and the considerable preliminary work necessary.

Presented as a poster presentation at the Thirty-second Annual Meeting of the Western Thoracic Surgical Association, Sun Valley, Idaho, June 21-24, 2006.

Received for publication July 27, 2006; revisions received Nov 15, 2006; accepted for publication Dec 18, 2006.

Address for reprints: Edward J. Hickey, MD, CHSS Data Center, Hospital for Sick Children, 555 University Ave, Toronto, Ontario M5G 1X8, Canada (E-mail: hickeydoc@yahoo.com).

J Thorac Cardiovasc Surg 2007;133:1588-96 $0022-5223 / \$ 32.00$

Copyright $\odot 2007$ by The American Association for Thoracic Surgery

doi:10.1016/j.jtcvs.2006.12.056
Objective: Delayed preconditioning genetically reprograms the response to ischemic injury. Subclinical bacterial lipopolysaccharide acts through preconditioning, powerfully protecting against experimental stroke. We investigated the potential for lipopolysaccharide to protect against brain injury related to cardiopulmonary bypass.

Methods: Neonatal piglets were blindly and randomly preconditioned with lipopolysaccharide $(n=6)$ or saline $(n=6)$. Three days later, they experienced 2 hours of deep hypothermic circulatory arrest before being weaned and supported anesthetized for 20 hours in an intensive care setting. Controls included cardiopulmonary bypass without deep hypothermic circulatory arrest $(\mathrm{n}=3)$ and no cardiopulmonary bypass $(\mathrm{n}=3)$. Brain injury was quantified by light and fluorescent microscopy (Fluoro-Jade; Histo-Chem, Inc, Jefferson, Ark).

Results: All animals were clinically indistinguishable before surgery. Perioperative and postoperative parameters between experimental groups were similar. No control animal scored falsely positive. Histologic scores were $0.33 \pm 0.21,0.66 \pm 0.42$, and $0.5 \pm 0.24$ in the cortex, basal ganglia, and hippocampus, respectively, in the lipopolysaccharide-treated animals but significantly worse in all saline control animals $(1.33 \pm 0.21, P<.01 ; 1.66 \pm 0.33, P=.09$; and $6.0 \pm 1.5, P<.01)$. One lipopolysaccharide-treated brain was histologically indistinguishable from controls.

Conclusion: This is the first evidence that lipopolysaccharide can precondition against cardiopulmonary bypass-related injury. Because lipopolysaccharide preconditioning is a systemic phenomenon offering proven protection against myocardial, hepatic, and pulmonary injury, this technique offers enormous potential for protecting against systemic neonatal injury related to cardiopulmonary bypass.

$\mathrm{P}$ reconditioning describes a concept whereby brief exposure to a harmful stimulus in a dose below the threshold for tissue injury provides robust protection against, or tolerance to, the injurious effects of a subsequent more severe insult. ${ }^{1}$ Two forms of preconditioning exist—acute and delayed—which are fundamentally different in nature. Acute preconditioning occurs within minutes and provides protection for a number of hours. It has been extensively studied, especially in the context of myocardial injury. Delayed preconditioning offers a far greater window of protection. The protective effects begin after 24 to 48 hours but continue for as long as 7 to 14 days. $^{2}$ Delayed preconditioning of the brain is a widely established experimental phenomenon in rodent models of regional and global ischemia, but this concept has not previously been applied to ischemic brain injury resulting during cardiopulmonary bypass (CPB).

A widely recognized trigger for delayed preconditioning is bacterial lipopolysaccharide (LPS) and its derivatives. Systemically administered in extremely low 


$$
\begin{aligned}
& \text { Abbreviations and Acronyms } \\
& \begin{aligned}
\text { CPB } & =\text { cardiopulmonary bypass } \\
\text { DHCA } & =\text { deep hypothermic circulatory arrest } \\
\text { LPS } & =\text { lipopolysaccharide } \\
\text { TLR } & =\text { Toll-like receptor } \\
\text { TNF } & =\text { tumor necrosis factor }
\end{aligned}
\end{aligned}
$$

doses, it provides protection against a variety of subsequent insults, including myocardial, renal, liver, and cerebral ischemia, where infarct volume can be reduced by as much as $60 \% .^{2}$ Evidence has recently emerged suggesting that delayed preconditioning involves a complete reprogramming at the genetic level, to provide systemic protection against both ischemic injury ${ }^{3}$ and activation of components of the cellular immune response. ${ }^{4}$ This concept, therefore, is extremely appealing in the context of ischemic CPB-related injury, where systemic inflammation, hypoxia-ischemia, and reperfusion injury act synergistically to cause end-organ damage.

Because LPS preconditioning consistently induces robust protection against ischemic brain injury in experimental rodents, we entertained the hypothesis that low-dose LPS may confer cerebral protection against ischemic injury sustained during periods of neonatal deep hypothermic circulatory arrest (DHCA). Using a piglet model of DHCA, involving a 20-hour reperfusion period in a fully monitored intensive care environment, we aimed to test this hypothesis by histologically quantifying regional brain injury after animals were blindly and randomly inoculated before surgery with either a preconditioning dose of LPS or saline placebo.

\section{Materials and Methods \\ Experimental Design}

Twelve neonatal piglets were inoculated intravenously, randomly, and blindly, with either normal saline (saline control group) or 20 $\mu \mathrm{g} / \mathrm{kg}$ Escherichia coli bacterial LPS (serotype 0111:B4, SigmaAldrich Corp, St Louis, Mo) (LPS group). Inoculation was performed under brief general anesthesia (1\% isofluorane). After recovery, they were observed closely for the first 24 hours for adverse signs, including sickness, lethargy, or sepsis. Three days (72 hours) later, animals were placed on full open-chested CPB and subjected to 2 hours of DHCA at $18^{\circ} \mathrm{C}$. After rewarming and separation from $\mathrm{CPB}$, the animals were supported anesthetized and ventilated with full invasive monitoring for a further 20 hours (warm reperfusion period) before being perfusion-fixed. Brain tissue was extracted for histologic and fluoroscopic (Fluoro-Jade; Histo-Chem Inc, Jefferson, Ark) scoring of neurologic injury in the frontal lobe cortex, basal ganglia, hippocampus, and cerebellum. Histologic controls included nonischemic controls $(n=3)$ subjected to 2 hours of deep hypothermic full-flow CPB with 20 hours of postbypass reperfusion and experimental shams $(n=3)$ anes- thetized and instrumented but not placed on $\mathrm{CPB}$, humanely killed 20 hours later.

\section{Surgical Procedures}

All animal experiments were conducted with the approval of the institution's Animal Care and Use Committee. The animals received humane care in compliance with the "Guide for the Care and Use of Laboratory Animals" published by the National Institutes of Health (NIH publication No. 85-23, revised 1995).

The surgical protocol, including CPB circuit and prime, was similar to that described previously by our laboratory. ${ }^{5}$ In brief, after anesthetic induction (1\% isofluorane) and placement of peripheral lines and temperature probes, surgical tracheotomy was performed to allow controlled ventilation, maintaining arterial oxygen and carbon dioxide tensions within normal limits.

The heart was exposed via a median sternotomy. After systemic heparinization (500 IU/kg), the aortic root and right atrial appendage were cannulated and CPB was established at 100 to $150 \mathrm{~mL}$. $\mathrm{kg}^{-1} \cdot \min ^{-1}$ to maintain mean arterial pressures of $50 \mathrm{~mm} \mathrm{Hg}$. The animal was then perfusion-cooled with a $\mathrm{pH}$-stat strategy to $18^{\circ} \mathrm{C}$ over 30 minutes. CPB was then ceased and the circulatory volume drained into the reservoir to induce uninterrupted circulatory arrest for 120 minutes. During this period, myocardial protection was afforded by slowly perfusing through an isolate coronary circulation. An aortic crossclamp was applied to the ascending aorta immediately distal to the aortic cannula, and soft clips were applied to the inferior and superior venae cavae, thereby isolating the coronary circulation through which cold blood was administered at a rate of 5 to $10 \mathrm{~mL} / \mathrm{min}$. Full-flow CPB was later re-established and the animal rewarmed, with the administration of carbonate $(8.4 \%)$ to correct acidosis. The lungs were ventilated and the animals separated from CPB in conjunction with a continuous infusion of intravenous norepinephrine $(2 \mathrm{~mL} / \mathrm{h})$ and volume replacement as necessary.

\section{Postoperative Care}

Protamine was administered (5 mg/1000 IU heparin), drains and bladder catheters were positioned, and all wounds were closed. Animals were then supported in a laboratory intensive care, anesthetized (intravenous propofol 3-5 $\mathrm{mL} / \mathrm{h}$ ), and ventilated, with fully alarmed invasive monitoring and continuous veterinary support. The arterial pressure was maintained with a mean greater than $50 \mathrm{~mm} \mathrm{Hg}$. After 20 hours of reperfusion, the animals were exsanguinated, irrigated ( $3 \mathrm{~L}$ of saline), and perfusion-fixed ( $2 \mathrm{~L}$ of ice-cold $4 \%$ paraformaldehyde). The brains were retrieved intact before being sectioned coronally, embedded in paraffin blocks, and 5 - $\mu \mathrm{m}$ slices mounted on slides for staining with hematoxylin and eosin or Fluoro-Jade.

\section{Staining with Fluoro-Jade}

Fluoro-Jade is an anionic fluorochrome that selectively stains irreversibly injured neurons with greater sensitivity and specificity than hematoxylin and eosin. ${ }^{6}$ After deparaffinization, slides were immersed for 20 minutes in $0.06 \%$ potassium permanganate before being washed several times in deionized water. Fluoro-Jade crystals were dissolved to a $0.0004 \%$ solution in $0.01 \%$ acetic acid and the slides were immersed for 20 minutes before thorough air-drying in a warm incubator. After xylene washes, slides 
TABLE 1. Experimental CPB parameters for the two experimental groups (LPS-preconditioned and saline control [unpreconditioned])

\begin{tabular}{|c|c|c|c|}
\hline & Saline control & LPS & $P$ value \\
\hline Weight (kg) & $2.8 \pm .18$ & $3.3 \pm .50$ & .43 \\
\hline Hematocrit (\%) & $28.5 \pm 1.9$ & $27.8 \pm 1.9$ & .81 \\
\hline $\mathrm{pH}$ & $7.36 \pm .04$ & $7.39 \pm .64$ & .64 \\
\hline Base deficit $(\mathrm{mmol} / \mathrm{L})$ & $-5.3 \pm 2.1$ & $-2.0 \pm 2.4$ & .33 \\
\hline $\mathrm{PaO}_{2}(\mathrm{~mm} \mathrm{Hg})$ & $245 \pm 39$ & $308 \pm 47$ & .35 \\
\hline $\mathrm{PaCO}_{2}(\mathrm{~mm} \mathrm{Hg})$ & $33 \pm 5.1$ & $36 \pm 3.3$ & .68 \\
\hline MAP (mm Hg) & $45.0 \pm 2.3$ & $50.8 \pm 4.4$ & .30 \\
\hline Heart rate (beats/min) & $117 \pm 5.4$ & $131 . \pm .1$ & .08 \\
\hline Core temp $\left({ }^{\circ} \mathrm{C}\right)$ & $37.3 \pm .32$ & $36.7 \pm .58$ & .54 \\
\hline Cooling duration (min) & $27.5 \pm 2.5$ & $25.8 \pm 2.0$ & .61 \\
\hline Rewarm duration (min) & $52.5 \pm 5.1$ & $50.0 \pm 4.1$ & .71 \\
\hline Total CPB duration (min) & $201 \pm 4.6$ & $195 \pm 4.6$ & .38 \\
\hline $\begin{array}{l}\text { Base deficit at } 25^{\circ} \mathrm{C} \text { during } \\
\text { rewarm (mmol/L) }\end{array}$ & $-13.8 \pm 2.2$ & $-11 \pm 1.3$ & .33 \\
\hline $\mathrm{HCO}_{3}(\mathrm{~mL})$ & $21 \pm 2.0$ & $17 \pm .3$ & .31 \\
\hline $\mathrm{pH}$ at wean & $7.41 \pm .03$ & $7.42 \pm .05$ & .95 \\
\hline $\begin{array}{l}\text { Base deficit at wean } \\
(\mathrm{mmol} / \mathrm{L})\end{array}$ & $-3.2 \pm 1.9$ & $-3.7 \pm 2.0$ & .86 \\
\hline $\mathrm{PaO}_{2}$ at wean $(\mathrm{mm} \mathrm{Hg})$ & $316 \pm 48$ & $245 \pm 56$ & .38 \\
\hline $\mathrm{PaCO}_{2}$ at wean $(\mathrm{mm} \mathrm{Hg})$ & $29 \pm 3.7$ & $28 \pm 4.6$ & .85 \\
\hline Hematocrit at wean $(\%)$ & $34 \pm 1.7$ & $32 \pm 2.5$ & .65 \\
\hline
\end{tabular}

$C P B$, Cardiopulmonary bypass; $L P S$, lipopolysaccharide.

were covered and viewed with an epifluorescent microscope with blue (450-490 nm) excitation light. A barrier filter allowing passage of all wavelengths longer than $515 \mathrm{~nm}$ results in a green emission color from cells stained positively with Fluoro-Jade.

\section{Histologic Scoring}

Slides were examined randomly by investigators blinded to the experimental protocol. Morphologic criteria used to identify ischemic neurons were a prominent eosinophilic nucleus, with lack of a well-defined nucleolus and Nissl substance, together with nuclear fragmentation and formation of dark apoptotic bodies. ${ }^{7}$ These are established signs of irreversible neuronal injury and avoid falsepositive identification of hyperchromatic neuronal artifact. ${ }^{7}$ Qualitative grading scores were applied according to the cerebral cortex, basal ganglia, hippocampus, and cerebellum (where $0=$ normal and $4=$ confluent, severe injury). The hippocampus is know to exhibit regional vulnerability, and therefore subregions (CA1, CA2, CA3, CA4, and superficial, middle, and deep dentate gyrus) were selectively scored, inasmuch as repeated studies with our model have demonstrated reproducible hierarchical injury within these areas.

\section{Statistical Analysis}

All data are expressed as mean \pm standard error of the mean. The unpaired Student $t$ tests were used to compare perioperative parameters and quantitative histologic scores between animals in the saline and LPS preconditioned groups or otherwise analysis of variance to compare values between groups with SAS statistical software (SAS, Inc, Cary, NC). Statistical significance was tested to the $95 \%$ confidence limit.

\section{Results \\ Perioperative Parameters}

All animals survived the experiment. Preoperatively, animals were indistinguishable; none exhibited signs of ill health or required veterinary intervention. The preoperative and CPB parameters of the two experimental groups (LPS and saline control) are given in Table 1. Postoperative parameters during the recovery period are provided in Table 2.

\section{Histologic Scores of Injury}

Histologic scores for each animal in the representative regions and subregions are given in Table 3 and Figure 1. Importantly, no false-positive scoring for injury occurred in any specimen from a sham or nonischemic control animal. All animals in the saline control group exhibited histologic evidence of ischemic injury in the cortex, basal ganglia, and especially the hippocampus. The regional vulnerability within the hippocampus is interesting, because conventionally the large CA1 neurons are regarded as the most sensitive to ischemic injury in the mammalian brain. Our laboratory consistently observes the pattern of hippocampal injury seen herein-the superficial region of the dentate gyrus is the most sensitive to injury, and with progressively more severe injury, ischemic neurons are then noted proportionately deeper within the dentate and then into the (adjacent) CA4. Similar dentate gyrus susceptibility has been noted by others ${ }^{8} 24$ to 48 hours after ischemia. No injury was detected in the cerebellum of any animal, even in the large Purkinje neurons.

Every animal preconditioned with LPS displayed significantly reduced histologic injury in the cortex and hippocampal subregions, regardless of whether scores were considered cumulatively or not. In fact, animal No. 5 in the LPS-preconditioned group exhibited no evidence of ischemic injury, such that specimens from all its respective brain regions were indistinguishable from those of nonischemic control and sham animals.

Fluoroscopic assessment with Fluoro-Jade reiterated the changes observed by light microscopy (Table 4 and Figure 2). Fluoro-Jade is highly sensitive and specific for irreversible neuronal injury and makes identification of positive neurons straightforward (Figure 3). Every brain region examined in this study except the cerebellum exhibited significantly lesser injury by Fluoro-Jade assessment after LPS preconditioning. No false-positive scores were observed in either the nonischemic control or sham animals.

In summary, both by light microscopic and by fluoroscopic criteria, LPS-preconditioned animals displayed sig- 
TABLE 2. Postoperative variables

\begin{tabular}{|c|c|c|c|c|c|c|}
\hline Group & After wean & 4 hours & 8 hours & 12 hours & 16 hours & 20 hours \\
\hline \multicolumn{7}{|c|}{ Mean arterial pressure (invasive) $(\mathrm{mm} \mathrm{Hg})$} \\
\hline Saline & $48.8 \pm 4.8$ & $48.8 \pm 4.8$ & $53.3 \pm 4.3$ & $57.4 \pm 3.1$ & $50 \pm 3.5$ & $51 \pm 3.2$ \\
\hline LPS & $57.8 \pm 6.4$ & $53.5 \pm 5.4$ & $64 \pm 4.4$ & $60 \pm 5.1$ & $56 \pm 3.7$ & $56 \pm 4.8$ \\
\hline$P$ & .29 & .48 & .12 & .68 & .27 & .43 \\
\hline \multicolumn{7}{|c|}{ Heart rate (beats/min) } \\
\hline Saline & $137 \pm 6$ & $143 \pm 8$ & $150 \pm 9$ & $154 \pm 6$ & $166 \pm 5$ & $166 \pm 4$ \\
\hline LPS & $127 \pm 7$ & $142 \pm 7$ & $157 \pm 5$ & $165 \pm 6$ & $163 \pm 9$ & $152 \pm 7$ \\
\hline$P$ & .32 & .99 & .54 & .30 & .79 & .16 \\
\hline \multicolumn{7}{|c|}{$\mathrm{Po}_{2}(\mathrm{~mm} \mathrm{Hg})$} \\
\hline Saline & $298 \pm 57$ & $217 \pm 43$ & $215 \pm 24$ & $173 \pm 33$ & $192 \pm 32$ & $241 \pm 17$ \\
\hline LPS & $237 \pm 32$ & $225 \pm 39$ & $149 \pm 9.8$ & $209 \pm 17$ & $249 \pm 27$ & $268 \pm 53$ \\
\hline$P$ & .41 & .89 & .03 & .37 & .28 & .64 \\
\hline \multicolumn{7}{|c|}{$\mathrm{PCO}_{2}(\mathrm{~mm} \mathrm{Hg})$} \\
\hline Saline & $34 \pm 2.6$ & $43.3 \pm 3.4$ & $37.5 \pm 3.1$ & $43.0 \pm 3.7$ & $35.6 \pm 2.3$ & $36.0 \pm 2.3$ \\
\hline LPS & $36 \pm 4.4$ & $43.0 \pm 3.2$ & $45 \pm 1.3$ & $47.5 \pm 4.5$ & $41.0 \pm 2.2$ & $33.0 \pm 5.2$ \\
\hline$P$ & .64 & .94 & .04 & .49 & .08 & .51 \\
\hline \multicolumn{7}{|l|}{$\mathrm{pH}$} \\
\hline Saline & $7.31 \pm .03$ & $7.32 \pm .03$ & $7.43 \pm .03$ & $7.43 \pm .05$ & $7.47 \pm .02$ & $7.47 \pm .02$ \\
\hline LPS & $7.33 \pm .02$ & $7.31 \pm .03$ & $7.36 \pm .03$ & $7.37 \pm .04$ & $7.45 \pm .04$ & $7.53 \pm .05$ \\
\hline$P$ & .67 & .81 & .16 & .40 & .66 & .32 \\
\hline \multicolumn{7}{|c|}{ Base excess (mmol/L) } \\
\hline Saline & $-7.5 \pm .8$ & $-5.1 \pm .9$ & $+0.9 \pm .8$ & $+2.9 \pm .9$ & $+2.9 \pm 1.9$ & $+3.9 \pm 1.2$ \\
\hline LPS & $-3.9 \pm 1.4$ & $-3.9 \pm 2.5$ & $-0.2 \pm 2.3$ & $+2.7 \pm 2.1$ & $+4.9 \pm 1.8$ & $+5.6 \pm 2.6$ \\
\hline$P$ & .06 & .66 & .66 & .94 & .38 & .55 \\
\hline \multicolumn{7}{|c|}{ Hematocrit (\%) } \\
\hline Saline & $32.1 \pm 1.3$ & $28.8 \pm .9$ & $31.2 \pm 2.1$ & $32.2 \pm 1.9$ & $36 \pm 1.4$ & $34 \pm 1.3$ \\
\hline LPS & $31.3 \pm 1.4$ & $30.8 \pm 2.4$ & $31.6 \pm 1.7$ & $34.2 \pm 1.7$ & $36 \pm 3.4$ & $32.5 \pm 3.1$ \\
\hline$P$ & .79 & .56 & .89 & .31 & .92 & .70 \\
\hline \multicolumn{7}{|c|}{ Temperature $\left({ }^{\circ} \mathrm{C}\right)$} \\
\hline Saline & $36.0 \pm .3$ & $36.7 \pm .5$ & $37.0 \pm .6$ & $37.2 \pm .4$ & $37.3 \pm .6$ & $37.2 \pm .23$ \\
\hline LPS & $36.4 \pm .5$ & $37.1 \pm .7$ & $37.9 \pm .4$ & $36.9 \pm .7$ & $37.8 \pm .4$ & $37.1 \pm .5$ \\
\hline$P$ & .50 & .60 & .31 & .68 & .56 & .85 \\
\hline
\end{tabular}

$P$ values compare animals between experimental groups inoculated with either lipopolysaccharide (LPS) or saline control.

nificantly reduced ischemic neuronal injury over their counterparts inoculated with saline placebo.

\section{Discussion}

This study describes the first successful application of delayed preconditioning with LPS in a model of CPB-related ischemic brain injury. In contrast to neuroprotective strategies targeting clinical stroke, which lacks a well-defined pre-aura, preconditioning against CPB-related ischemic brain injury is a far more applicable concept. In fact, CPB involving periods of circulatory arrest is the only circumstance in which a (controlled) severe global ischemic hit is routinely administered to the brain in a premeditated fashion.

Cerebral preconditioning is not a new idea. Several acute preconditioning techniques are widely established experimentally, in particular brief hypoxia-ischemia (or hypoxia mimetics ${ }^{9}$ ). It is surprising, therefore, that these techniques were tested against CPB-related brain injury only recently. ${ }^{9,10}$ The initial application to CPB by Yeh and associates, ${ }^{10}$ involved the use of acute ischemic preconditioning. By contrast, delayed preconditioning is a considerably more appealing concept, not least because it offers a significantly greater window of protection and potency. ${ }^{2}$ In clinical terms, for example, a 7-day window of tolerance would span the entire operative and intensive care periods, during which numerous compounding factors are believed to influence progression of the initial injury.

Although several agents, including ischemia-hypoxia (and their mimetics ${ }^{9}$ ), can induce delayed protection, LPS and its derivatives are among the most closely studied. LPS preconditioning was originally noted to confer protection against subsequent lethal doses of LPS (homologous tolerance). ${ }^{11}$ However, it also exhibits cross-tolerance, where a nonlethal stress confers tolerance to a subsequent stress that is different in nature from the first. Thus, systemic LPS has 
TABLE 3. Individual histologic scores assigned to animals in the respective experimental groups by light microspcopy, including controls

\begin{tabular}{|c|c|c|c|c|c|c|c|c|c|c|c|c|}
\hline \multirow[b]{2}{*}{ Animal } & \multirow[b]{2}{*}{ Cortex } & \multirow{2}{*}{$\begin{array}{c}\text { Basal } \\
\text { ganglia }\end{array}$} & \multirow[b]{2}{*}{ Cerebellum } & \multicolumn{7}{|c|}{ Hippocampus } & \multirow{2}{*}{$\begin{array}{l}\text { HCamp } \\
\text { total }\end{array}$} & \multirow{2}{*}{$\begin{array}{c}\text { Cumul } \\
\text { total }\end{array}$} \\
\hline & & & & CA1 & CA2 & CA3 & CA4 & DG sup & DG med & DG deep & & \\
\hline \multicolumn{13}{|l|}{ Saline } \\
\hline 1 & 1 & 1 & 0 & 0 & 0 & 1 & 2 & 3 & 2 & 0 & 8 & 10 \\
\hline 2 & 2 & 2 & 0 & 0 & 0 & 3 & 3 & 3 & 1 & 1 & 11 & 15 \\
\hline 3 & 1 & 1 & 0 & 0 & 0 & 1 & 1 & 2 & 1 & 0 & 5 & 7 \\
\hline 4 & 1 & 1 & 0 & 0 & 0 & 0 & 0 & 0 & 0 & 0 & 0 & 2 \\
\hline 5 & 1 & 2 & 0 & 0 & 0 & 0 & 1 & 3 & 1 & 0 & 5 & 8 \\
\hline 6 & 2 & 3 & 0 & 0 & 0 & 0 & 2 & 3 & 2 & 0 & 7 & 12 \\
\hline Mean & $1.33 \pm 0.21$ & $1.67 \pm 0.33$ & 0 & 0 & 0 & $0.83 \pm 0.48$ & $1.5 \pm 0.43$ & $2.33 \pm 0.5$ & $1.2 \pm 0.31$ & $0.17 \pm 0.17$ & $6.0 \pm 1.5$ & $9 \pm 1.8$ \\
\hline \multicolumn{13}{|c|}{ 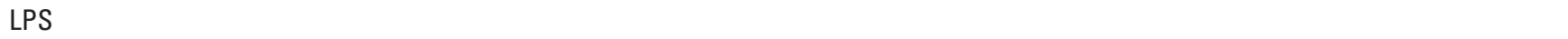 } \\
\hline 1 & 0 & 0 & 0 & 0 & 0 & 0 & 0 & 1 & 0 & 0 & 1 & 1 \\
\hline 2 & 1 & 0 & 0 & 0 & 0 & 0 & 0 & 0 & 0 & 0 & 0 & 1 \\
\hline 3 & 0 & 0 & 0 & 0 & 0 & 0 & 1 & 0 & 0 & 0 & 1 & 1 \\
\hline 4 & 1 & 2 & 0 & 0 & 0 & 0 & 0 & 1 & 0 & 0 & 1 & 4 \\
\hline 5 & 0 & 0 & 0 & 0 & 0 & 0 & 0 & 0 & 0 & 0 & 0 & 0 \\
\hline 6 & 0 & 2 & 0 & 0 & 0 & 0 & 0 & 0 & 0 & 0 & 0 & 2 \\
\hline Mean & $0.33 \pm 0.21$ & $0.67 \pm 0.42$ & 0 & 0 & 0 & 0 & $0.17 \pm 0.17$ & $.33 \pm .21$ & 0 & 0 & $0.5 \pm 0.22$ & $1.5 \pm 0.6$ \\
\hline $\begin{array}{l}P, \text { LPS vs } \\
\text { saline }\end{array}$ & $>.007$ & .09 & - & - & - & .11 & .016 & .004 & .004 & .34 & .005 & .003 \\
\hline \multicolumn{13}{|l|}{$\begin{array}{c}\text { Nonischemic } \\
\text { control }\end{array}$} \\
\hline 1 & 0 & 0 & 0 & 0 & 0 & 0 & 0 & 0 & 0 & 0 & 0 & 0 \\
\hline 2 & 0 & 0 & 0 & 0 & 0 & 0 & 0 & 0 & 0 & 0 & 0 & 0 \\
\hline 3 & 0 & 0 & 0 & 0 & 0 & 0 & 0 & 0 & 0 & 0 & 0 & 0 \\
\hline \multicolumn{13}{|l|}{ Sham } \\
\hline 1 & 0 & 0 & 0 & 0 & 0 & 0 & 0 & 0 & 0 & 0 & 0 & 0 \\
\hline 2 & 0 & 0 & 0 & 0 & 0 & 0 & 0 & 0 & 0 & 0 & 0 & 0 \\
\hline 3 & 0 & 0 & 0 & 0 & 0 & 0 & 0 & 0 & 0 & 0 & 0 & 0 \\
\hline
\end{tabular}

Cumulative hippocampal (HCampal total) and overall cumulative (Cumul total) scores are indicated in the far right columns. LPS, Lipopolysaccharide; DG, dentate gyrus; sup, superficial.

Histologic score (H\&E)

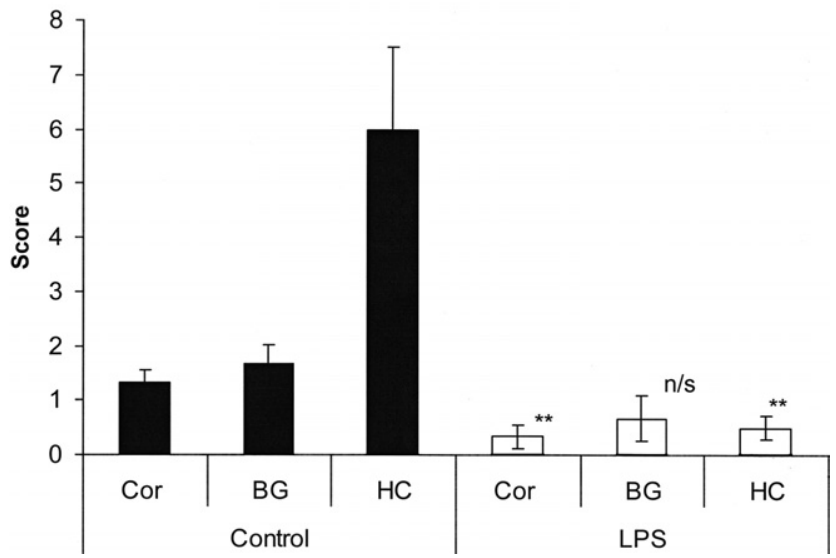

Figure 1. Graphic illustration of the histologic injury in frontal lobe cortex, basal ganglia, and hippocampus occurring in the LPS-preconditioned and saline control groups, as determined by routine light microscopy. ${ }^{* *} P<.01 ; n / s$, not significant. Cor, Cortex; $B G$, basal ganglia; $H C$, hippocampus; LPS, lipopolysaccharide. now been shown to also induce ischemic tolerance in several systems including the liver, myocardium, kidney, and brain. ${ }^{11-13}$ In fact, the characteristics of LPS preconditioning appear to share several hallmark features with delayed ischemic preconditioning in the brain, ${ }^{11}$ suggesting that common pathways may link the numerous primary preconditioning stimuli.

Rodent models generating large infarct zones consistently demonstrate reduction of injury by as much as $60 \%$ after systemic LPS inoculation. ${ }^{2}$ Histologic models of injury after DHCA instead require extended ischemic periods to generate scattered evidence of ischemic injury., ${ }^{9,14,15}$ These extended durations of DHCA represent a departure from clinical practice and are therefore a significant limitation in the experimental study of DHCA. Nevertheless, whereas all nonpreconditioned animals in this present study displayed brain injury in all regions other than cerebellum, brain regions from some of the preconditioned animals were indistinguishable from control groups, with no ischemic injury apparent, suggesting a robust protective effect. The 
TABLE 4. Individual histologic scores assigned to animals in the respective experimental groups by Fluoro-Jade including controls

\begin{tabular}{|c|c|c|c|c|c|c|c|c|c|c|c|c|}
\hline \multirow[b]{2}{*}{ Animal } & \multirow[b]{2}{*}{ Cortex } & \multirow{2}{*}{$\begin{array}{c}\text { Basal } \\
\text { ganglia }\end{array}$} & \multirow[b]{2}{*}{ Cerebellum } & \multicolumn{7}{|c|}{ Hippocampus } & \multirow{2}{*}{$\begin{array}{c}\text { HCamp } \\
\text { total }\end{array}$} & \multirow{2}{*}{$\begin{array}{c}\text { Cumul } \\
\text { total }\end{array}$} \\
\hline & & & & CA1 & CA2 & CA3 & CA4 & DG sup & DG med & DG deep & & \\
\hline \multicolumn{13}{|l|}{ Saline } \\
\hline 2 & 2 & 1 & 0 & 0 & 0 & 0 & 2 & 3 & 2 & 0 & 7 & 10 \\
\hline 3 & 2 & 3 & 0 & 0 & 0 & 0 & 1 & 3 & 2 & 0 & 6 & 11 \\
\hline 4 & 0 & 2 & 0 & 0 & 0 & 0 & 0 & 1 & 0 & 0 & 1 & 3 \\
\hline 6 & 2 & 3 & 0 & 0 & 0 & 0 & 1 & 3 & 2 & 0 & 6 & 11 \\
\hline Mean & $1.5 \pm 0.34$ & $2.33 \pm 0.33$ & 0 & 0 & 0 & $0.33 \pm 0.21$ & $1.33 \pm 0.33$ & $2.67 \pm 0.33$ & $1.67 \pm 0.33$ & 0 & $6.0 \pm 1.1$ & $9.8 \pm 1.4$ \\
\hline \multicolumn{13}{|c|}{ 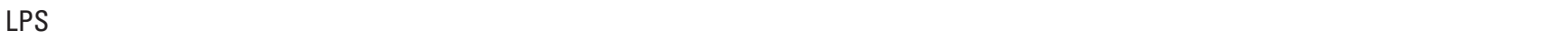 } \\
\hline 1 & 1 & 1 & 0 & 0 & 0 & 0 & 0 & 2 & 1 & 0 & 3 & 5 \\
\hline 2 & 0 & 0 & 0 & 0 & 0 & 0 & 0 & 0 & 0 & 0 & 0 & 0 \\
\hline 3 & 1 & 1 & 0 & 0 & 0 & 0 & 0 & 1 & 0 & 0 & 1 & 3 \\
\hline $\begin{array}{c}P, \text { LPS vs } \\
\text { saline }\end{array}$ & $>.034$ & .002 & - & - & - & .15 & .011 & .002 & .007 & - & .003 & .002 \\
\hline \multicolumn{13}{|l|}{$\begin{array}{c}\text { Nonischemic } \\
\text { control }\end{array}$} \\
\hline 1 & 0 & 0 & 0 & 0 & 0 & 0 & 0 & 0 & 0 & 0 & 0 & 0 \\
\hline 2 & 0 & 0 & 0 & 0 & 0 & 0 & 0 & 0 & 0 & 0 & 0 & 0 \\
\hline 3 & 0 & 0 & 0 & 0 & 0 & 0 & 0 & 0 & 0 & 0 & 0 & 0 \\
\hline \multicolumn{13}{|l|}{ Sham } \\
\hline 1 & 0 & 0 & 0 & 0 & 0 & 0 & 0 & 0 & 0 & 0 & 0 & 0 \\
\hline 2 & 0 & 0 & 0 & 0 & 0 & 0 & 0 & 0 & 0 & 0 & 0 & 0 \\
\hline 3 & 0 & 0 & 0 & 0 & 0 & 0 & 0 & 0 & 0 & 0 & 0 & 0 \\
\hline
\end{tabular}

Cumulative hippocampal (HCampal total) and overall cumulative (Cumul total) scores are indicated in the far right columns. LPS, Lipopolysaccharide; DG, dentate gyrus; sup, superficial; med, medium.

\section{Fluoro-Jade score}

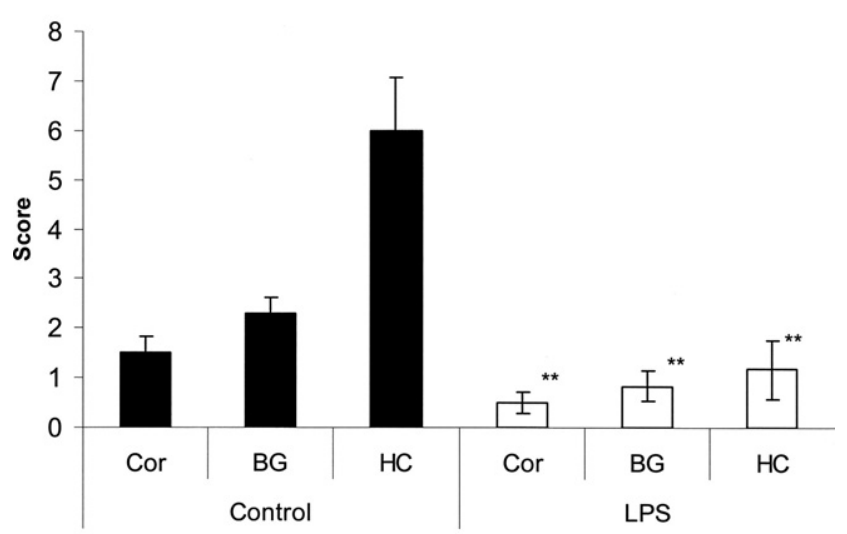

Figure 2. Graphic illustration of the histologic injury in frontal lobe cortex, basal ganglia, and hippocampus occurring in the LPS-preconditioned and saline control groups, as determined by FluoroJade staining. ${ }^{* *} P<$. 01 . Cor, Cortex; $B G$, basal ganglia; HC, hippocampus; LPS, lipopolysaccharide. premise on which exaggerated animal DHCA models are used is that observed protection should translate to some degree of protection against clinical DHCA injury, however slight. In addition, the protection demonstrated in this present study is important for several reasons: (1) LPS preconditioning has never been tested in conjunction with CPB; (2) although descriptions of LPS preconditioning exist in swine, ${ }^{16}$ none have examined cerebral protection; and (3) conflicting reports exist as to whether or not the neonatal mammalian brain is amenable to preconditioning. ${ }^{17-19}$

\section{Conclusions}

Considerable energy has recently been spent elucidating the mechanisms underlying preconditioning. Acute and delayed forms are fundamentally different. Acute preconditioning probably involves posttranslational processing and may have several features in common with myocardial preconditioning, including the role of nitric oxide, ${ }^{20}$ adenosine A1 receptors, ${ }^{21}$ and adenosine triphosphate-dependent potassium channels. ${ }^{21}$ By contrast, delayed preconditioning is an 


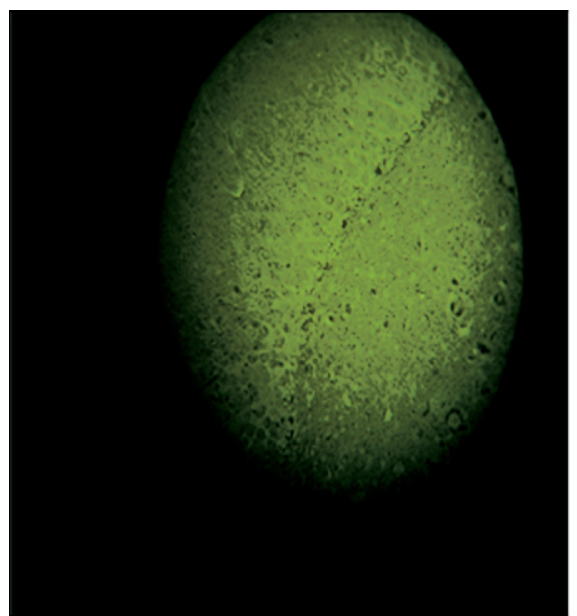

LPS preconditioned

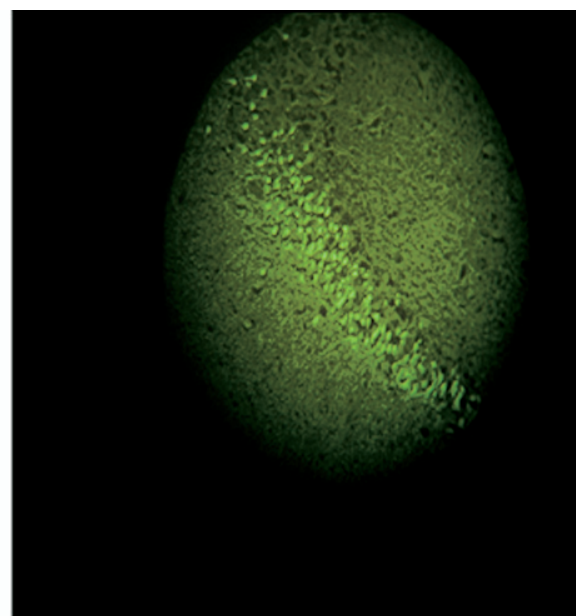

Non-preconditioned

Figure 3. Representative images of hippocampal (dentate gyrus) sections from LPS-preconditioned and saline control (unpreconditioned ) brains stained with Fluoro-Jade as viewed through a fluorescent microcope. The positive cells are clearly discernible, littering the granular cell layer of the dentate. LPS, Lipopolysaccharide.

active process. It involves an endogenous program of neuroprotection $^{22,23}$ featuring a complex cascade of intracellular signaling events leading to new synthesis of proteins ${ }^{23,24}$ that ultimately reprograms the cellular response to subsequent injury.

LPS is a ligand for Toll-like receptor (TLR)-4. ${ }^{25}$ TLRs are evolutionary historic transmembrane proteins that recognize generic constituents of foreign bacteria (doublestranded RNA, unmethylated DNA sequences, and LPSs) to trigger downstream inflammatory pathways. In addition, however, it is recognized that injured and necrotic cells also release endogenous ligands, including RNA, chromosomal DNA, heat shock proteins, and components of extracellular matrix. ${ }^{26}$ TLRs may therefore represent the common pathway linking diverse initiators of delayed preconditioning.

One theory proposed ${ }^{26}$ is that a very low level of stimulation of TLRs (either through exogenous LPS or endogenous components generated through a preconditioning stimulus such as subinjurious ischemia), together with proinflammatory cytokines (tumor necrosis factor- $\alpha$ [TNF$\alpha$ ], interleukin-1), results in downstream generation of both proinflammatory and anti-inflammatory cytokines. In addition, suppressors of inflammation are produced, which include decoy receptors and decoy ligands. During the second stimulation, these decoys and anti-inflammatory cytokines predominate, resulting in an overall reduction in tissue injury. Alternatively, if the initial preconditioning stimulus exceeds beyond a threshold dose, the initial response is overwhelmed by pro-inflammatory processes, and the preconditioning response will not occur. Thus, preconditioning is similar to a "trip switch." If the preconditioning dose falls above the threshold, the trip is flipped, and tissue injury will occur instead.

The above hypothesis would explain several conundrums, including the paradox as to why an obligatory step in the delayed preconditioning process is the generation of potentially harmful inflammatory mediators. TNF- $\alpha$ in particular, and its downstream signaling mediator ceramide, are critical effectors of LPS-induced neuroprotection. ${ }^{27,28}$ LPS treatment accompanied by concomitant neutralizing antiTNF- $\alpha$ reverses the protective effect of LPS preconditioning. ${ }^{11}$ Further downstream, the effector pathways are less clear, but one suggestion that is gathering support involves a central role of interferon- $\beta$, secondary to the TNF-signaling axis (reviewed in reference 1). A neuroprotective role of interferon- $\beta$ is supported by the recent finding that its systemic administration improves stroke outcome. ${ }^{29}$

The precise cascade of intracellular signaling events is clearly complex. However, the end result appears to be an entire genetic reprogramming of the genomic response to injury, which shifts the balance of outcome from cell death to cell survival. ${ }^{3}$ Microarray genome survey analysis suggests this genetic switch mimics the pattern seen in hibernation. ${ }^{3}$ Thus, as in hibernation, preconditioning elicits endogenous genetic adaptations that confer tolerance to the injurious effects of oxygen deprivation.

Of particular interest is the effect of delayed preconditioning on aspects of the inflammatory response, ${ }^{4}$ because inflammatory processes are increasingly being recognized as crucial propagators of ischemic brain injury. Rodent 
studies have recently demonstrated that although the total cellular infiltrate into the infarct zone is not different after preconditioning, the number of activated neutrophils is significantly reduced, as determined by reduced $\mathrm{CD} 11 \mathrm{~b}$ expression. ${ }^{4}$ The notion that delayed preconditioning might alter the profile of the systemic inflammatory response is of huge significance to CPB-related injury and clearly is an exciting area for future study.

The application to functional models represents another avenue for future investigation. We chose to use a model quantifying histologic neuronal injury because this remains a frequently used and reliable index of ischemic brain injury. Fluoro-Jade is especially sensitive for injured neurons, greatly facilitating their detection. Others have instead used markers of apoptosis or even functional outcome. Although difficult and crude in swine, functional models represent the proof of concept that we are intending to pursue in future studies. Microarray genome survey has additionally proved a valuable tool for both quantifying response to injury and providing insight into the putative mechanisms of preconditioning, ${ }^{3}$ and we are actively pursuing this technique in swine models at present.

One obstacle to the clinical application of LPS preconditioning is the risk posed by administering a potentially lethal substance to clinically vulnerable patients, irrespective of the tiny doses necessary. Interestingly, specific ligands for individual TLR subtypes have been shown to display cross-tolerance. ${ }^{30}$ For example, lipoteichoic acid preferentially binds TLR-2 and will induce tolerance to LPS (TLR-4) and visa versa. Future efforts should therefore explore the possibility that additional less noxious TLR ligands can be identified that confer similar protection to LPS through TLR cross-tolerance.

\section{References}

1. Hertzog PJ, O'Neill LA, Hamilton JA. The interferon in TLR signaling: more than just antiviral. Trends Immunol. 2003;24:534-9.

2. Hill JK, Gunion-Rinker L, Kulhanek D, Lessov N, Kim CS, Clark WM, et al. Temporal modulation of cytokine expression following focal cerebral ischemia in mice. Brain Res. 1999;820:45-54.

3. Stenzel-Poore MP, Stevens SL, Xiong Z, Lessov NS, Harrington CA, Mori $\mathrm{M}$, et al. Effect of ischaemic preconditioning on genomic response to cerebral ischaemia: similarity to neuroprotective strategies in hibernation and hypoxia-tolerant states. Lancet. 2003;362:1028-37.

4. Rosenzweig HL, Lessov NS, Henshall DC, Minami M, Simon RP, Stenzel-Poore MP. Endotoxin preconditioning prevents cellular inflammatory response during ischemic neuroprotection in mice. Stroke. 2004;35:2576-81.

5. Schultz JM, Karamlou T, Swanson J, Shen I, Ungerleider R. Hypothermic low-flow cardiopulmonary bypass impairs pulmonary and right ventricular function more than circulatory arrest. Ann Thorac Surg. 2006;81:474-80.

6. Schmued L, Albertson C, Slikker W. Fluoro-Jade: a novel fluorochrome for the sensitive and reliable histochemical localization of neuronal degeneration. Brain Res. 1997;751:37-46.

7. Cammermeyer J. The importance of avoiding the "dark" neurons in experimental neuropathology. Acta Neuropathol. 1961;1:245-70.

8. Martin LJ, Sieber FE, Traystman RJ. Apoptosis and necrosis occur in separate neuronal populations in hippocampus and cerebellum after ischemia and are associated with differential alterations in metabotropic glutamate receptor signaling pathways. J Cereb Blood Flow Metab. 2000;20:153-67.

9. Kerendi F, Halkos ME, Kin H, Corvera JS, Brat DJ, Wagner MB, et al. Upregulation of hypoxia inducible factor is associated with attenuation of neuronal injury in neonatal piglets undergoing deep hypothermic circulatory arrest. J Thorac Cardiovasc Surg. 2005; 130:1079.

10. Yeh CH, Wang YC, Wu YC, Lin YM, Lin PJ. Ischemic preconditioning or heat shock pretreatment ameliorates neuronal apoptosis following hypothermic circulatory arrest. J Thorac Cardiovasc Surg. 2004; 128:203-10.

11. Tasaki K, Ruetzler CA, Ohtsuki T, Martin D, Nawashiro H, Hallenbeck JM. Lipopolysaccharide pre-treatment induces resistance against subsequent focal cerebral ischemic damage in spontaneously hypertensive rats. Brain Res. 1997;748:267-70.

12. Fernandez ED, Flohe S, Siemers F, Nau M, Ackermann M, Ruwe M, et al. Endotoxin tolerance protects against local hepatic ischemia/ reperfusion injury in the rat. $J$ Endotoxin Res. 2000;6:321-8.

13. Dawson DA, Furuya K, Gotoh J, Nakao Y, Hallenbeck JM. Cerebrovascular hemodynamics and ischemic tolerance: lipopolysaccharideinduced resistance to focal cerebral ischemia is not due to changes in severity of the initial ischemic insult, but is associated with preservation of microvascular perfusion. J Cereb Blood Flow Metab. 1999;19: 616-23.

14. Shum-Tim D, Nagashima M, Shinoka T, Bucerius J, Nollert G, Lidov HG, et al. Postischemic hyperthermia exacerbates neurologic injury after deep hypothermic circulatory arrest. J Thorac Cardiovasc Surg. 1998;116:780-92.

15. Ye J, Yang L, Del Bigio MR, Filgueiras CL, Ede M, Summers R, et al. Neuronal damage after hypothermic circulatory arrest and retrograde cerebral perfusion in the pig. Ann Thorac Surg. 1996;61: 1316-22.

16. Klaus S, Heringlake M, Block K, Nolde J, Staubach K, Bahlmann L. Metabolic changes detected by microdialysis during endotoxin shock and after endotoxin preconditioning. Intensive Care Med. 2003;29: 634-41.

17. Tuor UI, Del Bigio MR, Chumas PD. Brain damage due to cerebral hypoxia/ischemia in the neonate: pathology and pharmacological modification. Cerebrovasc Brain Metab Rev. 1996;8:159-93.

18. Zhao P, Zuo Z. Prenatal hypoxia-induced adaption and neuroprotection that is inducible nitric oxide-synthase dependent. Neurobiol Dis. 2005;20:871-80.

19. Eklind S, Mallard C, Arvidsson P, Hagberg H. Lipopolysaccharide induces both a primary and a secondary phase of sensitization in the developing rat brain. Pediatr Res. 2005;58:112-6.

20. Gidday JM, Shah AR, Maceren RG, Wang Q, Pelligrino DA, Holtzman DM, et al. Nitric oxide mediates cerebral ischemic tolerance in a neonatal rat model of hypoxic preconditioning. J Cereb Blood Flow Metab. 1999;19:331-40.

21. Heurteaux C, Lauritzen I, Widmann C, Lazdunski M. Essential role of adenosine, adenosine A1 receptors, and ATP-sensitive $\mathrm{K}+$ channels in cerebral ischemic preconditioning. Proc Natl Acad Sci U S A. 1995; 92:4666-70

22. Nandagopal K, Dawson TM, Dawson VL. Critical role for nitric oxide signaling in cardiac and neuronal ischemic preconditioning and tolerance. J Pharmacol Exp Ther. 2001;297:474-8.

23. Chen J, Graham SH, Zhu RL, Simon RP. Stress proteins and tolerance to focal cerebral ischemia. J Cereb Blood Flow Metab. 1996;16: 566-77.

24. Bordet R, Deplanque D, Maboudou P, Puisieux F, Pu Q, Robin E, et al. Increase in endogenous brain superoxide dismutase as a potential mechanism of lipopolysaccharide-induced brain ischemic tolerance. J Cereb Blood Flow Metab. 2000;20:1190-6.

25. Wietek C, Miggin SM, Jefferies CA, O'Neill LA. Interferon regulatory factor-3-mediated activation of the interferon-sensitive response element by Toll-like receptor (TLR) 4 but not TLR3 requires the p65 subunit of NF-kappa. J Biol Chem. 2003;278:50923-31.

26. Kariko K, Weissman D, Welsh FA. Inhibition of toll-like receptor and cytokine signaling - a unifying theme in ischemic tolerance. J Cereb Blood Flow Metab. 2004;24:1288-304. 
27. Nawashiro H, Martin D, Hallenbeck JM. Neuroprotective effects of TNF binding protein in focal cerebral ischemia. Brain Res. 1997;778: 265-71.

28. Furuya K, Ginis I, Takeda H, Chen Y, Hallenbeck JM. Cell permeable exogenous ceramide reduces infarct size in spontaneously hypertensive rats supporting in vitro studies that have implicated ceramide in induction of tolerance to ischemia. J Cereb Blood Flow Metab. 2001;21:226-32.
29. Liu H, Xin L, Chan BP, Teoh R, Tang BL, Tan YH. Interferon-beta administration confers a beneficial outcome in a rabbit model of thromboembolic cerebral ischemia. Neurosci Lett. 2002;327: 146-8.

30. Dalpke AH, Lehner MD, Hartung T, Heeg K. Differential effects of CpG-DNA in Toll-like receptor-2/-4/-9 tolerance and cross-tolerance. Immunology. 2005;116:203-12. 